\title{
Impact of off-shell dynamics on the transport properties and the dynamical evolution of charm quarks at RHIC and LHC temperatures
}

\author{
Maria Lucia Sambataro ${ }^{1,2, a}$, Salvatore Plumari ${ }^{1,2, b}$, Vincenzo Greco $^{1,2}$ \\ ${ }^{1}$ Department of Physics and Astronomy 'E. Majorana', University of Catania, Via S. Sofia 64, 95125 Catania, Italy \\ ${ }^{2}$ Laboratori Nazionali del Sud, INFN-LNS, Via S. Sofia 62, 95123 Catania, Italy
}

Received: 8 June 2020 / Accepted: 24 November 2020 / Published online: 11 December 2020

(C) The Author(s) 2020

\begin{abstract}
We evaluate drag and diffusion transport coefficients comparing a quasi-particle approximation with onshell constituents of the QGP medium and a dynamical quasiparticles model with off-shell bulk medium at finite temperature T. We study the effects of the width $\gamma$ of the particles of the bulk medium on the charm quark transport properties exploring the range where $\gamma<M_{q, g}$. We find that offshell effects are in general quite moderate and can induce a reduction of the drag coefficient at low momenta that disappear already at moderate momenta, $p \gtrsim 2-3 \mathrm{GeV}$. We also observe a moderate reduction of the breaking of the fluctuation-dissipation theorem (FDT) at finite momenta. Moreover, we have performed a first study of the dynamical evolution of HQ elastic energy loss in a bulk medium at fixed temperature extending the Boltzmann (BM) collision integral to include off-shell dynamics. A comparison among the Langevin dynamics, the BM collisional integral with on-shell and the BM extension to off-shell dynamics shows that the evolution of charm energy when off-shell effects are included remain quite similar to the case of the on-shell BM collision integral.
\end{abstract}

\section{Introduction}

Heavy quarks (HQs), namely charm and bottom, are considered as a solid probe to characterize the matter created in the QGP phase [1-8]. The large mass of heavy quarks has several implications in this context. They are produced in the early stage of the collisions by pQCD process and being $M_{H Q} \gg T$ also the thermal pair production and annihilation processes are negligible. The HQ propagation through the QGP medium can be described as a diffusion

\footnotetext{
a e-mail: sambataro@lns.infn.it

b e-mail: salvatore.plumari@dfa.unict.it (corresponding author)
}

process assimilated to a Brownian motion due to their large masses and perturbatice interaction leading to collisions with small momentum transfer [2,3,9]. Furthermore, the large mass has the effect to reduce the equilibration rate of heavy quarks in the medium relative to their light counterparts leading to a thermalization time comparable to the one of the life time of the fireball $[3,10]$. Therefore, the standard approach to describe the propagation of HQ in QGP has been quite often treated within the framework of the FokkerPlanck equation [2,11-21]. However, the evidence of nonperturbative interaction and the large initial temperatures at LHC, $M_{c} \simeq 3 T \simeq\left\langle p_{\text {bulk }}\right\rangle$, hint at a scattering dynamics more appropriately described by a Boltzmann collision integral that implies significant deviations from a gaussian fluctuation around the average momentum of the charm quark [9,22-29].

One of the main observable for HQs that it has been also extensively used as a probe of QGP, is the nuclear suppression factor, $R_{A A}\left(p_{T}\right)$, [30-32]. It is defined as the ratio between the heavy flavor hadrons produced in nucleusnucleus collisions with respect to those produced in protonproton collisions. Another observable extensively studied is the elliptic flow [33,34], $v_{2}\left(p_{T}\right)=\langle\cos (2 \phi)\rangle$, a measure of the anisotropy in the angular distribution of heavy mesons in momentum space, as a response to the initial anisotropy in coordinate space in non-central collisions. In literature several studies have been performed in these years, to study theoretically both these observables with the aim to understand heavy quark dynamics in QGP employing the Langevin or the on-shell Boltzmann transport equation $[9,12,13,22,25,29,35-44]$. However, being the QGP strongly interacting, a full quantum description of the charm quark interaction should include in principle also the off-shell dynamics, an approach that has been developed only in [45] for the study of the transport coefficients and it is included 
in the PHSD approach to heavy-ion collisions $[27,38,46]$. Recently, it has been shown that also off-shell effects can play a role in the hadronization processes [47]. In this paper we extended this study exploring also the effects of larger widths and in particular discussing the effect also in terms of the fluctuations dissipation theorem (FDT). Moreover, we present a first study of the time evolution of the charm momentum in a bulk medium at fixed temperature $\mathrm{T}$ comparing directly the Langevin evolution, the Boltzmann on-shell evolution and an extension of the Boltzmann collision integral to include off-shell dynamics. We also discuss the impact that off-shell dynamics can have on the $R_{A A}\left(p_{T}\right)$.

The paper is organized as follows. In the next sections we will briefly present the on-shell Boltzmann transport equation, the Fokker-Planck (Langevin) one and the definition of drag and diffusion coefficients in both on-shell and off-shell approaches. In Sect. 3, we discuss the results obtained for the transport coefficients in both on-shell and off-shell models. Section 4 is devoted to discuss the dynamical evolution of charm quarks in a bulk medium at finite $\mathrm{T}$ by comparing the results obtained in Langevin, on-shell and off-shell Boltzmann approaches. Section 5 contains the summary and conclusions.

\section{Boltzmann transport equation and transport coefficients}

In this section we are interested to study both the transport coefficients and the time evolution of the phase-space distribution function of heavy quarks (HQs). The starting point in the study of propagation of heavy quark is the relativistic transport equation for HQs scattering in a bulk medium of quarks and gluons. We therefore briefly describe the relativistic Boltzmann-Vlasov equation from which we will deduce the transport coefficients for on-shell dynamics and the Fokker-Planck equation. The on-shell transport equation can be expressed by the Boltzmann-Vlasov equation given by the following integro-differential equation:

$$
\left\{p^{\mu} \partial_{\mu}+m^{*}(x) \partial_{\mu} m^{*}(x) \partial_{p}^{\mu}\right\} f_{Q}(x, p)=C\left[\hat{f}_{q}, \hat{f}_{g}, f_{Q}\right]
$$

where $f_{Q}(x, p)$ and $\hat{f}_{q, g}(x, p)$ are the phase-space distribution functions for the heavy quark and light quarks and gluons respectively, while $C\left[f_{q}, f_{g}, f_{Q}\right](x, p)$ is the relativistic Boltzmann-like collision integral allowing to describe the short range interaction between heavy quark and particles of plasma. The distribution function of the bulk medium of quarks and gluons has in general to be determined by another set of equations that could be the Boltzmann-Vlasov equations for quark and gluons [48-50]. In the present study, we want to address a direct comparison between two different dynamics: the relativistic Langevin dynamics and the rela- tivistic Boltzmann transport theory. In the second approach, we will discuss the role of on-shell and off-shell effects on the HQ dynamics. In order to have a better focusing and testing the dynamics between these different approaches the bulk medium will be considered as a thermal bath at equilibrium at some temperature $T$. Moreover, we will calculate the different transport coefficients of HQs in a static medium at finite temperature. This will give the response of the medium to the propagation of HQs under fixed thermodynamical conditions. This is a first step before studying the more complex case of the expanding medium in realistic uRHIC where gradients of density and temperature are involved. Therefore in our calculations we neglect effects caused by space-time variation of the scalar mean fields, $\partial_{\mu} m^{*}(x) \approx 0$. Assuming that the distribution function is $x$ independent, i.e. the plasma is uniform, each variation of the distribution function is due to collisions and the Boltzmann equation is simplified to a integro-differential equation only respect to time:

$p^{0} \partial_{0} f_{Q}=C\left[\hat{f}_{q}, \hat{f}_{g}, f_{Q}\right]$.

We will consider only two-body collisions where the collision integral $C\left[f_{q}, f_{g}, f_{Q}\right](p)$ can be expressed by the following relation:

$$
\begin{aligned}
C[f]= & \frac{1}{2 E_{p}} \int \frac{d^{3} \mathbf{q}}{2 E_{q}(2 \pi)^{3}} \int \frac{d^{3} \mathbf{q}^{\prime}}{2 E_{q^{\prime}}(2 \pi)^{3}} \int \frac{d^{3} \mathbf{p}^{\prime}}{2 E_{p^{\prime}}(2 \pi)^{3}} \\
& \left.\cdot \frac{1}{d_{Q}} \sum_{g, q, \bar{q}} \mid \mathcal{M}(g(q, \bar{q}), Q \rightarrow g(q, \bar{q}), Q)\right)\left.\right|^{2} \\
& \cdot(2 \pi)^{4} \delta^{4}\left(p+q-p^{\prime}-q^{\prime}\right) \\
& {\left[f_{Q}\left(\mathbf{p}^{\prime}\right) \hat{f}\left(\mathbf{q}^{\prime}\right)-f_{Q}(\mathbf{p}) \hat{f}(\mathbf{q})\right] }
\end{aligned}
$$

where $\mathbf{p}(\mathbf{q})$ and $\mathbf{p} /(\mathbf{q} /)$ represent respectively the initial and final momentum of heavy quark (plasma particle), $\left|\mathcal{M}_{Q}\right|^{2}$ is the squared modulus of scattering matrix of the process. In order to solve the collision integral it is necessary to evaluate the scattering matrix $\left|\mathcal{M}_{Q}\right|^{2}$. In our calculations the HQs interact with the medium by mean of two-body collisions regulated by the scattering matrix of the processes $g+Q \rightarrow$ $g+Q$ and $q(\bar{q})+Q \rightarrow q(\bar{q})+Q$.

A successful way to treat non-perturbative effects in heavy-quark scattering is given by quasi-particle approach (QPM), in which the interaction is encoded in the quasiparticle masses that behave like massive constituents of free gas plus a background field interaction given by a temperature dependent bag constant, for details see Ref. [51]. The main feature of QPM approach is that the resulting coupling is significantly stronger than the one coming from $p Q C D$ running coupling, particularly at $T \rightarrow T_{C}$. It has been shown that QPM can reproduce the lattice QCD equation of state: pressure, energy density and interaction measure $T_{\mu}^{\mu}=\epsilon-3 P$. 
The relations of the masses of light quarks and gluons to the coupling and temperature are calculated in a perturbative approach:

$$
\begin{aligned}
& m_{g}^{2}=\frac{1}{6} g(T)^{2}\left[\left(N_{c}+\frac{1}{2} N_{f}\right) T^{2}+\frac{N_{c}}{2 \pi^{2}} \sum_{q} \mu_{q}^{2}\right], \\
& m_{u, d, s}^{2}=\frac{N_{c}^{2}-1}{8 N_{c}} g(T)^{2}\left[T^{2}+\frac{\mu_{u, d, s}^{2}}{\pi^{2}}\right],
\end{aligned}
$$

where $N_{f}$ and $N_{c}$ are respectively the number of flavours and colours, $\mu_{q}$ is the chemical potential of the q flavour that in our calculation is neglected. Even if the formal relation is perturbative-like, the $g(T)$ is obtained by a fit to the energy density of lattice QCD (IQCD) and it is expressed by:

$g^{2}(T)=\frac{48 \pi^{2}}{\left[\left(11 N_{c}-2 N_{f}\right) \ln \left[\lambda\left(\frac{T}{T_{c}}-\frac{T_{s}}{T_{c}}\right)\right]\right]^{2}}$,

where $\lambda=2.6$ and $T_{s} / T_{c}=0.57$, with $T_{c}=155 \mathrm{MeV}$. We obtain a non perturbative behaviour of the coupling especially for $T \rightarrow T_{c}$.

The evaluation of the scattering matrix has been performed considering the leading-order diagrams. In this approach the effective coupling $g(T)$ leads to effective vertices and a dressed massive gluon propagator for $g+Q \rightarrow g+Q$ and massive quark propagator for $q(\bar{q})+Q \rightarrow q(\bar{q})+Q$ scatterings. The detail of the calculations can be found in Ref. [45].

\subsection{On-shell transport coefficients and Fokker-Planck equation}

We give a brief description of the derivation of HQ transport coefficients. We can also express the collision integral in relation to the rate of collisions $\omega(\mathbf{p}, \mathbf{k})$ between HQ and light bulk particles, where $\mathbf{k}$ is the transferred momentum during the collision:

$C[f]=\int d^{3} \mathbf{k}[\omega(\mathbf{p}+\mathbf{k}, \mathbf{k}) f(\mathbf{p}+\mathbf{k})-\omega(\mathbf{p}, \mathbf{k}) f(\mathbf{p})]$.

The rate of collision $\omega(\mathbf{p}, \mathbf{k})$ is given by:

$\omega(\mathbf{p}, \mathbf{k})=d_{Q G P} \int \frac{d^{3} \mathbf{q}}{(2 \pi)^{3}} \hat{f}(\mathbf{q}) v_{q, p} \frac{d \sigma_{p, q \rightarrow p-k, q+k}}{d \Omega}$.

In this relation $d_{Q G P}$ defines degree of freedom of particle in collision with heavy quark, $v_{q, p}$ defines the relative velocity and $\sigma_{p, q \rightarrow p-k, q+k}$ is the differential cross section of the scattering process.
Differential cross section can be expressed by the following relation:

$$
\begin{aligned}
\frac{d \sigma_{p, q \rightarrow p-k, q+k}}{d \Omega}= & \frac{1}{(2 \pi)^{6}} \frac{1}{v_{p, q}} \frac{1}{2 E_{q}} \frac{1}{2 E_{p}} \frac{1}{d_{Q} d_{Q G P}} \\
& \times \sum\left|\mathcal{M}_{Q}\right|^{2} \frac{1}{2 E_{q+k}} \frac{1}{2 E_{p-k}}(2 \pi)^{4} \delta \\
& \left(E_{p}+E_{q}-E_{p-k}-E_{q+k}\right)
\end{aligned}
$$

with the $\sum$ intended over all the elastic scattering channels $g+Q \rightarrow g+Q$ and $q(\bar{q})+Q \rightarrow q(\bar{q})+Q$. The nonlinear integro-differential Boltzmann equation cannot be easily solved and a way to simplify the calculation is to employ the Landau approximation leading to a relativistic FokkerPlanck equation with momentum dependent transport equation. This assumption is physical motivated by the suggestion that during collision the transfer momentum $\mathbf{k}$ is small and we can operate an expansion of the integral:

$$
\begin{aligned}
f(\mathbf{p}+\mathbf{k}) \omega(\mathbf{p}+\mathbf{k}, \mathbf{k})= & f(\mathbf{p}) \omega(\mathbf{p}, \mathbf{k})+\mathbf{k} \frac{\partial}{\partial \mathbf{p}}(\omega f) \\
& +\frac{1}{2} k_{i} k_{j} \frac{\partial^{2}}{\partial p_{i} \partial p_{j}}(\omega f)+\cdots
\end{aligned}
$$

Defining the following quantities:

$$
\begin{aligned}
A_{i}(\mathbf{p}, T) & =\int d^{3} k k_{i} \omega(\mathbf{p}, \mathbf{k}) \\
B_{i, j}(\mathbf{p}, T) & =\frac{1}{2} \int d^{3} k k_{i} k_{j} \omega(\mathbf{p}, \mathbf{k})
\end{aligned}
$$

the collision integral $C[f]$ in Eq. 3 becomes:

$$
\frac{d f(\mathbf{p})}{d t}=\frac{\partial}{\partial p_{i}}\left[A_{i}(\mathbf{p}, T) f(\mathbf{p})+\frac{\partial}{\partial p_{j}}\left[B_{i, j}(\mathbf{p}, T) f(\mathbf{p})\right]\right] .
$$

The Eq. 11 is the Fokker-Planck equation and the quantities defined by the Eq. 10 are the drag and diffusion coefficients of the propagation of HQ in the thermal bath at temperature T. If we consider an isotropic medium, we can express the drag and diffusion coefficients by the following relations:

$$
\begin{aligned}
A_{i}(\mathbf{p}, T) & =A(p, T) p_{i} \\
B_{i, j}(\mathbf{p}, T) & =B_{L}(p, T) P_{i, j}^{\|}(\mathbf{p})+B_{T}(p, T) P_{i, j}^{\perp}(\mathbf{p}) .
\end{aligned}
$$

The diffusion coefficient is expressed by a longitudinal $B_{L}$ and a transversal $B_{T}$ component respect to the HQ momentum where $P_{i, j}^{\|}(\mathbf{p})=p_{i} p_{j} / \mathbf{p}^{2}$ and $P_{i, j}^{\perp}(\mathbf{p})=\delta_{i, j}-$ $\left(p_{i} p_{j} / \mathbf{p}^{2}\right)$ are the projection operators on the longitudinal and transverse momentum components. Using the definition in Eq. 10 we get the following expression for $A_{i}$ :

$$
\begin{aligned}
& A_{i}(\mathbf{p}, T) \\
& =\frac{1}{2 E_{p}} \int \frac{d^{3} \mathbf{q}}{2 E_{q}(2 \pi)^{3}} \int \frac{d^{3} \mathbf{q}^{\prime}}{2 E_{q^{\prime}}(2 \pi)^{3}} \int \frac{d^{3} \mathbf{p}^{\prime}}{2 E_{p^{\prime}}(2 \pi)^{3}}
\end{aligned}
$$




$$
\begin{aligned}
& \times \frac{1}{d_{Q}} \times \sum\left|\mathcal{M}_{Q}\right|^{2}(2 \pi)^{4} \delta^{4}\left(p+q-p^{\prime}-q^{\prime}\right) \\
& \times \hat{f}(q)\left[\left(p-p^{\prime}\right)_{i}\right] \equiv\left\langle\left\langle\left(p-p^{\prime}\right)_{i}\right\rangle\right\rangle .
\end{aligned}
$$

while for $B_{i, j}$ :

$B_{i, j}(\mathbf{p}, T)=\frac{1}{2}\left\langle\left\langle\left(p-p^{\prime}\right)_{i}\left(p^{\prime}-p\right)_{j}\right\rangle\right\rangle$.

Finally, drag, transverse and longitudinal diffusion coefficients can be calculated as follows:

$$
\begin{aligned}
B_{L}(p, T) & =\frac{1}{2} \frac{p_{i} p_{j}}{p^{2}} B_{i, j} \\
& =\frac{1}{2}\left[\left\langle\left\langle\mathbf{p}^{\prime} \cdot \mathbf{p}\right\rangle\right\rangle p^{2}-2\left\langle\left\langle\mathbf{p}^{\prime} \cdot \mathbf{p}\right\rangle\right\rangle+p^{2}\langle\langle\mathbf{1}\rangle\rangle\right] \\
B_{T}(p, T) & =\frac{1}{2}\left[\delta_{i, j}-\frac{p_{i} p_{j}}{p^{2}}\right] B_{i, j} \\
& =\frac{1}{4}\left[\left\langle\left\langle p^{\prime 2}\right\rangle\right\rangle-\left\langle\left\langle\left(\mathbf{p} \cdot \mathbf{p}^{\prime}\right)^{2}\right\rangle\right\rangle / p^{2}\right] .
\end{aligned}
$$

and for drag coefficient:

$$
\begin{aligned}
A(p, T) & =p_{i} A_{i} / p^{2} \\
& =\langle\langle\mathbf{1}\rangle\rangle-\left\langle\left\langle\mathbf{p}^{\prime} \cdot \mathbf{p}\right\rangle\right\rangle / p^{2} .
\end{aligned}
$$

We recall that the standard approach to evaluate the quantities in Eqs. 13 and 14 is to write the integral in the c.m. frame using the c.m. scattering angles and the momentum $\mathbf{q}$ of the plasma particle:

$$
\begin{aligned}
\langle\langle F(\mathbf{p}, \mathbf{p}, T)\rangle\rangle= & \frac{1}{(2 \pi)^{3}} \int_{0}^{\infty} d \mathbf{q q}^{2} \int_{-1}^{+1} d \cos \alpha \\
& \times \int_{t_{\min }}^{t_{\max }} d t v_{\text {rel }} \frac{d \sigma}{d t} \hat{f}(\mathbf{q}) \int_{0}^{2 \pi} d \phi_{c m} F(\mathbf{p}, \mathbf{p}, T),
\end{aligned}
$$

where $\alpha$ is the polar angle of $\mathbf{q}$ and the Mandelstam variable $t$ is expressed in terms of momentum $\hat{\mathbf{p}}$ of heavy quark in the c.m. scattering system by $t=\left(p-p^{\prime}\right)^{2}=-2|\hat{\mathbf{p}}|^{2}(1-$ $\left.\cos \theta_{c m}\right)$. Finally, the differential cross section takes the form:

$$
\frac{d \sigma}{d t}=\frac{1}{16 \pi} \frac{1}{\left[\left(s-M_{Q}^{2}-m^{2}\right)^{2}-4 M_{Q}^{2} m^{2}\right]} \frac{1}{d Q} \sum\left|\mathcal{M}_{\mathcal{Q}}\right|^{2} .
$$

\subsection{Off-shell transport coefficients}

In order to have a more accurate description, the propagation of heavy quark can be also treated taking into account off-shell effects due to collisions with quasi-particle in the plasma. We want to investigate how the off-shell quantum effects modify the evolution of charm quark respect to the on-shell case that as a first approximation is commonly used to study the propagation of these particles in the bulk of light quarks and gluons. The collision integral, that in the on-shell case is expressed by Eq. 3, in the off-shell case can be written as:

$$
\begin{aligned}
C[f]= & \int d m_{i} S\left(m_{i}\right) \int d m_{f} S\left(m_{f}\right) \\
& \times \frac{1}{2 E_{p}} \int \frac{d^{3} \mathbf{q}}{2 E_{q}(2 \pi)^{3}} \int \frac{d^{3} \mathbf{q}^{\prime}}{2 E_{q^{\prime}}(2 \pi)^{3}} \int \frac{d^{3} \mathbf{p}^{\prime}}{2 E_{p^{\prime}}(2 \pi)^{3}} \\
& \times \frac{1}{\gamma_{Q}} \sum\left|\mathcal{M}_{Q}\right|^{2}(2 \pi)^{4} \delta^{4}\left(p+q-p^{\prime}-q^{\prime}\right) \\
& \times\left[f\left(\mathbf{p}^{\prime}\right) \hat{f}\left(\mathbf{q}^{\prime}, m_{f}\right)-f(\mathbf{p}) \hat{f}\left(\mathbf{q}, m_{i}\right)\right] .
\end{aligned}
$$

In the above equation $S\left(m_{i}\right)$ and $S\left(m_{f}\right)$ are the corresponding spectral functions for both initial and final quark and gluon masses. The spectral functions have been discussed later in Eq. 22. The dynamical quasi-particle model (DQPM) describes QCD properties in terms of the singleparticle Green's functions which leads to the description of QGP in terms of strongly interacting massive effective quasiparticles with broad spectral functions [52]. In this approach the parton masses and widths are determined by fitting the quasi-particle entropy density to the 1QCD entropy density reproducing the QCD equation of state extracted from lattice QCD calculations [53]. The aim of this study is an evaluation of the off-shell effects due to plasma quasi-particles. In the DQPM approach in Ref. [45], partons are dressed by non perturbative spectral function $S\left(q^{0}\right)$ which associates a spectrum of energies to a particle of momentum q. The ansatz used to model a nonzero width is obtained by replacing the free spectral function by a Lorentzian form [45]. As shown in Ref. [45] the Lorentzian form has a peak at small values of $p / T$ at the pole mass of the charm quarks and for it a non-relativistic approximation is a good approximation. We have to specify that in this work, due to the small values of the widths with respect to the charm quark mass, we consider only on-shell charm quarks that it should be by far a reliable approximation while for the light quarks and gluons we use the one from Eq. (21) that are exactly the ones used in Ref. [45].

In this work, we are interested in a non-relativistic approximation of partonic spectral function in which at small momenta $q^{0} \approx m$, in this way $S\left(q^{0}\right)$ is parametrized by a Breit-Wigner function $S^{B W}\left(m_{i}\right)$ [45,54]. The width of the partons in the perturbative limit are given by $\gamma \approx g^{2} T \ln g^{-1}$ where the physical process contributing to the functional form of the widths are elastic scattering like $g g \rightarrow g g$, $g q \rightarrow g q$ and $q q(\bar{q}) \rightarrow q q(\bar{q})$ are included. The functional forms of bulk particles widths $\gamma_{g}$ and $\gamma_{q}$ associated to spectral function for $\mu_{q}=0$ are given by:

$$
\begin{aligned}
& \gamma_{g}(T)=\frac{1}{3} N_{C} \frac{g^{2}\left(T / T_{C}\right)}{8 \pi} T \ln \left[\frac{2 c}{g^{2}\left(T / T_{C}\right)}+1\right] \\
& \gamma_{q}(T)=\frac{1}{3} \frac{N_{C}^{2}-1}{2 N_{C}} \frac{g^{2}\left(T / T_{C}\right)}{8 \pi} T \ln \left[\frac{2 c}{g^{2}\left(T / T_{C}\right)}+1\right]
\end{aligned}
$$




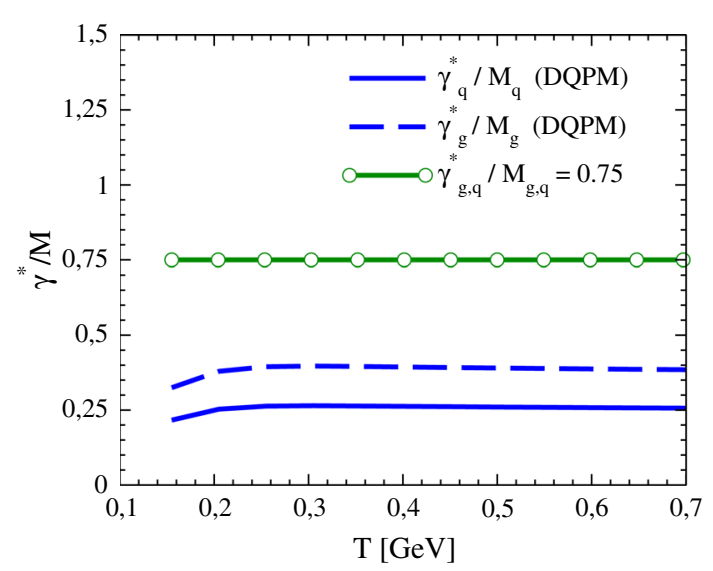

Fig. 1 Ratio between widths calculated in DQPM approach and the Pole Mass $M_{i}$ as function of temperature for quarks (blue solid line) and gluons (blue dashed line). The green solid line is the corresponding case with constant ratio fixed to $\gamma_{i}^{*} / M_{i}=0.75$

Fitting the entropy density on the 1QCD data, the constant $c$ is fixed to $c=14.4$. The spectral function associated to light quark and gluon in the plasma are expressed by:

$S_{i}^{B W}\left(m_{i}\right)=\frac{2}{\pi} \frac{m_{i}^{2} \gamma_{i}^{*}}{\left(m_{i}^{2}-M_{i}^{2}\right)^{2}+\left(m_{i} \gamma_{i}^{*}\right)^{2}}$

where $S_{i}^{B W}$ fulfills the normalization

$$
\int_{0}^{\infty} d m_{i} S_{i}\left(m_{i}, T\right)=1
$$

In Eq. 22, $M_{i}$ is the pole mass of gluon and light quark defined in Eq. 4 and $\gamma_{i}^{*}$ is the width associated to each particle mass. Such widths are related to $\gamma_{i}$ calculated in DQPM approach by the relation $2 q_{i}^{0} \gamma_{i}=m_{i} \gamma_{i}^{*}$ [45]. Since we are taking into account a regime where $\gamma<M_{i}$, the $\gamma_{i}^{*}$ of Eq. 22 can be written $\gamma_{i}^{*} \approx 2 \gamma_{i}$. The off-shell dynamics implies that the values of partonic masses can be different before and after scattering process, i.e. $m_{i} \neq m_{f}$, differently from onshell case in which $m_{i}=m_{f}$, where Breit-Wigner function becomes a delta function centered at Pole mass $M_{i}$. In Fig. 1, ratio between $\gamma_{i}^{*}$ and Pole mass $M_{i}$ are shown for gluon and light quark.

If we consider the off-shell quantum effects for the plasma particle, the quantity of Eq. 18 can be written:

$$
\begin{aligned}
& \left\langle\left\langle F\left(m_{i}, m_{f}, \mathbf{p}, \mathbf{p} /, T\right)\right\rangle\right\rangle=\int d m_{i} S\left(m_{i}\right) \int d m_{f} S\left(m_{f}\right) \\
& \times \frac{1}{(2 \pi)^{3}} \int_{0}^{\infty} d \mathbf{q q}^{2} \int_{-1}^{+1} d \cos \alpha \int_{t_{\min }}^{t_{\max }} d t v_{r e l} \\
& \times \frac{d \sigma}{d t} \hat{f}\left(m_{i}, \mathbf{q}\right) \int_{0}^{2 \pi} d \phi_{c m} F\left(m_{i}, m_{f}, \mathbf{p}, \mathbf{p} /, T\right)
\end{aligned}
$$

where $m_{i}$ and $m_{f}$ are respectively the initial and final mass of partons respectively. In this case the Mandelstam variable $t=\left(p-p^{\prime}\right)=2 M_{Q}^{2}-2 \hat{E}_{p} \hat{E}_{p^{\prime}}+2|\hat{\mathbf{p}}|\left|\hat{\mathbf{p}}^{\prime}\right| \cos \theta_{c m}$. We clarify that here we are keeping the couplig of quark and gluons to be the same in the on-shell and off-shell case to see the main direct effect of the inclusion of finite widths for the quasi-particles. Of course another approach could be to upscale $g(T)$ to have the same energy density in both onshell and off-shell case. Therefore, we have calculated the energy density of the system for both on-shell and off-shell approaches. The energy density for on-shell case is:

$\epsilon_{o n}=d_{Q G P} \int \frac{d^{3} \mathbf{q}}{(2 \pi)^{3}} \hat{f}(\mathbf{q}) E_{\mathbf{q}}$

while for the off-shell approach is the following

$\epsilon_{o f f}=d_{Q G P} \int d m_{i} S\left(m_{i}\right) \int \frac{d^{3} \mathbf{q}}{(2 \pi)^{3}} \hat{f}\left(m_{i}, \mathbf{q}\right) E_{\mathbf{q}}$.

keeping the same coupling constants we have seen a decrease of the energy density of the system for the off-shell case of about $10 \%$ for $\gamma_{D O P M}^{*}$ and $15 \%$ for $\gamma_{i}^{*} / M_{i}=0.75$ this change in energy density corresponds to a change of $g(T)$ by only few percent. We will discuss in the next Sections the impact on the transport coefficient that can be attibuited to differences in the energy density of the bulk matter and those that remain a pure off-shell effects.

\section{Results for transport coefficients: on-shell and off-shell}

In the following we will compare the results coming from the on-shell expression in Eq. 18 with the one in Eq. 23 that include the off-shell effects.

Before systematically study and compare the transport coefficients between the two different approaches presented in the previous sections we describe the common features in the following calculations. The number of thermal quark flavors is set to $n_{f}=3$, the medium temperature is kept fixed. A Boltzmann distribution is used for the thermal light flavor quark and gluon distribution. The charm quark mass is fixed to $M_{c}=1.3 \mathrm{GeV}$. To regulate the collinear divergence of the $\mathrm{t}$-channel in the scattering matrix, the following replacement is performed $1 / t \rightarrow 1 /\left(t-\mu_{D}^{2}\right)$. Where we have set the Debye screening mass to $m_{D}=\sqrt{4 \pi \alpha_{S}(T)} T=g(T) T$. In the off-shell case this replacement takes the form $1 / t \rightarrow$ $1 /\left(t-\mu_{D}^{2}+i 2 \gamma_{g}\left(p_{f}^{0}-p_{i}^{0}\right)\right)$ where $p^{0}$ is the energy of charm quark [45]. Numerically the drag and diffusion coefficients have been evaluated using a Monte-Carlo integration method for both on-shell and off-shell approach. We have studied the convergency of the integrals depending on the number of Monte-Carlo sampling $N_{s}$ and we have found that the most appropriate values are $N_{s}=5 \times 10^{6}$ for the on-shell case and 


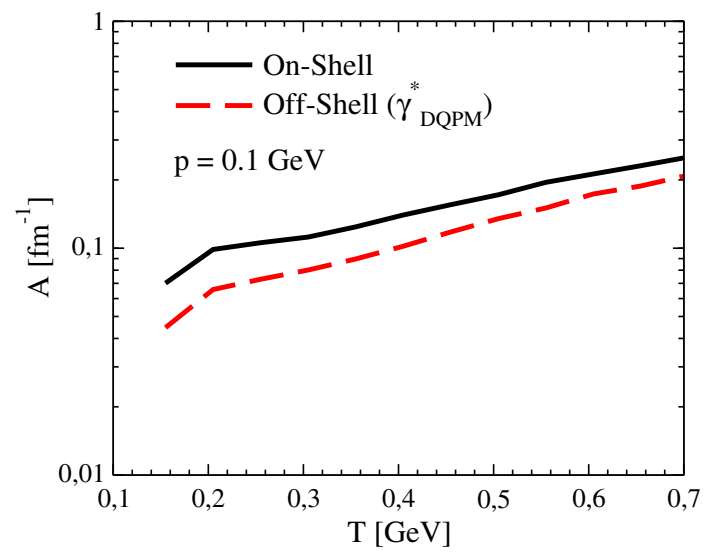

Fig. 2 Drag coefficient $(A(T))$ as a function of the medium temperature at fixed HQ momentum $p=0.1 \mathrm{GeV}$ for on-shell approach (black solid line) and off-shell approach (red dashed line)

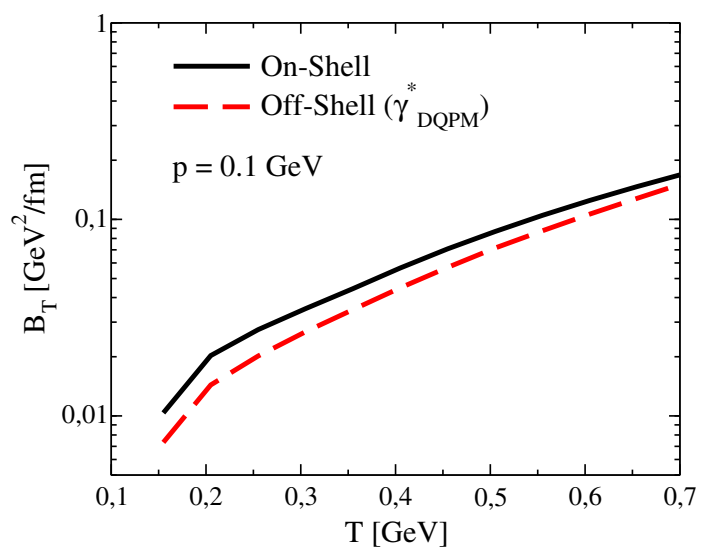

Fig. 3 Diffusion coefficient $B_{T}(T)$ as a function of the medium temperature at fixed HQ momentum $p=0.1 \mathrm{GeV}$ for on-shell approach (black solid line) and off-shell approach (red dashed line)

$N_{s}=3 \times 10^{8}$ for the off-shell one. In Figs. 2 and 3 we compare the transport coefficients as a function of the medium temperature with a fixed HQ momentum of $p=0.1 \mathrm{GeV} / \mathrm{c}$ for the two different approaches studied in this paper. Solid lines refers to on-shell calculations while red dashed lines for off-shell calculations. In Fig. 2 we show the results for the drag coefficient $A$. If quantum off-shell effects for bulk are considered the drag coefficients decrease of about $30 \%$ in the temperature regime of $T \sim 1-2 T_{c}$. Similar conclusion we get also for the diffusion coefficient $B_{T}$ as show in Fig. 3 , where in this case we observe a reduction of about $35 \%$ in the same range of temperature. Both difference decrease at increasing temperature.

We have checked if the decrease in the drag can be a mere effect of the decrease of the equilibrium energy density $\epsilon$. Therefore we have calculated the $A / \epsilon$ ratio as shown in Fig. 4 for different values of temperature. We can see that when we divide drag coefficient obtained in on-shell and off-shell mode by the respective values of the energy density of the

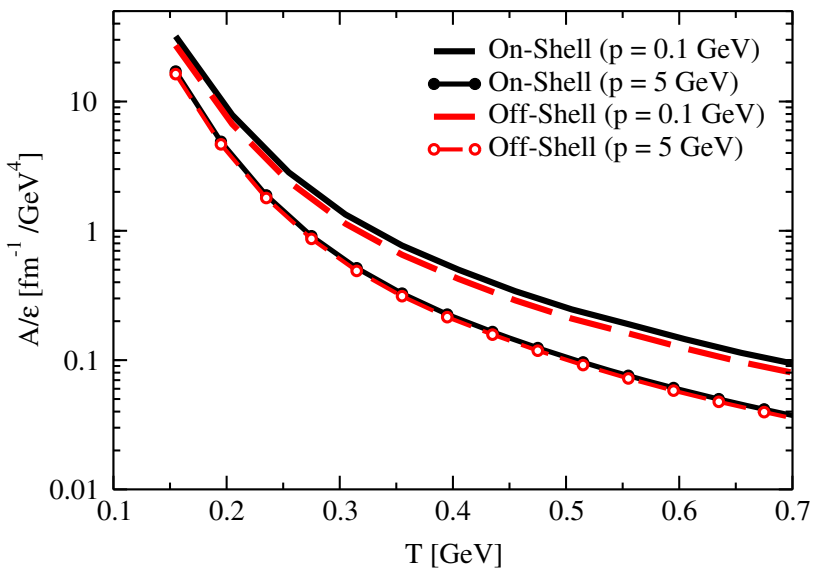

Fig. 4 Ratio between drag coefficient $(A)$ and energy density $(\epsilon)$ as function of temperature for two different HQ momentum $p=0.1 \mathrm{GeV}$ and $p=5 \mathrm{GeV}$. Black solid and red dashed line respectively for onshell and off-shell for $p=0.1 \mathrm{GeV}$ while full black circles and open red circles respectively for on-shell and off-shell for $p=5 \mathrm{GeV}$

bulk system, the decrease of coefficient in off-shell case is completely re-absorbed for intermediate and high momentum. The scaling with $\epsilon$ is only partially fulfilled in the limit $p \rightarrow 0$ and we see that a difference between on-shell and off-shell mode of about $10 \%$ remains even when the comparison is done renormalizing at the same energy density. This allows to draw a first conclusion about the fact that there is an impact of off-shell effects at low $p$, but a sizeable part can be traced-back to a change of the energy density when simply increasing increasing the width. Furthermore already at intermediate momenta $p$ the transport coefficients become the same once renormalized to the energy density, as we can see in Fig. 4 comparing open and filled circles. This is true at least when the values of the widths are relatively small as in DQPM approach. In this context, we want also to check the violation of fluctuation-dissipation theorem (FDT) [9] for on-shell and off-shell case. The validity of this relation can be verified evaluating the ratio between diffusion coefficient $B_{T}$, obtained by scattering matrix $\mathcal{M}_{Q}$ with the value of $B_{T}$ predicted by fluctuation-dissipation relation. In order to fulfill the FDT this ratio should be equal to 1 . In general, when we calculate transport coefficient with scattering matrix one obtains however a significant deviation [9]. In Fig. 5 it is shown the ratio between $B_{T}$ and $T E A$ where $E=\sqrt{p^{2}+M^{2}}$ for the two cases discussed in this paper for on-shell and off-shell partons. We observe that the FDT is better verified when off-shell bulk is taken into account where we obtain an improvement with respect to on-shell case of about $10 \%$. Moreover we observe that the FDT is better verified at higher temperature where we obtain a deviation lower than $15 \%$. In the results shown in the previous figures we have considered the widths $\gamma_{i}^{*}$ given by the DQPM approach [45]. As shown in Fig. 1, such widths (i.e. $\gamma_{g}^{*} \approx 260 \mathrm{MeV}$, 


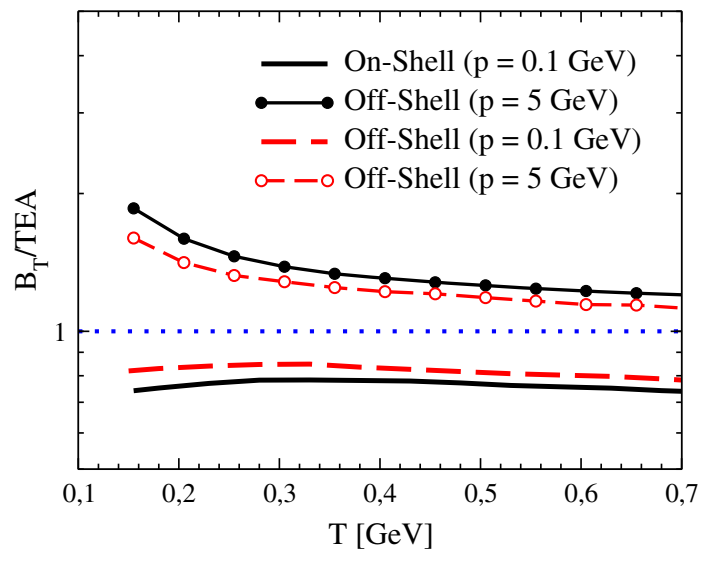

Fig. $5 B_{T} / T E A$ as a function of temperature for both on-shell and off-shell approaches and $p=0.1 \mathrm{GeV}$ and $p=5 \mathrm{GeV}$. Same legend as in Fig. 4

$\gamma_{q}^{*} \approx 110 \mathrm{MeV}$ at $T=200 \mathrm{MeV}$ ) are significant smaller than the quasi-particle masses.

In order to explore also the impact of quantum off-shell effects on the transport coefficients we artificially increase the widths considering $\gamma^{*}$ about 2-3 times larger than those of DQPM approach for quarks, i.e. $\gamma^{*} / M=0.75$ for both quarks and gluons (i.e. $\gamma_{g}^{*} \approx 520 \mathrm{MeV}, \gamma_{q}^{*} \approx 330 \mathrm{MeV}$ at $T=200 \mathrm{MeV}$ ) as shown by green line in Fig. 1. We consider larger widths with respect to the DQPM, because they are conceivable in other approaches especially considering values of the shear viscosity to entropy density ratio, $\eta / s$, is about 0.1 , while for DQPM it is stays in the range $\eta / s \sim$ $0.2-0.3$ for $T \sim T_{c}$.

In Figs. 6 and 7 we have shown the HQ transport coefficients, respectively drag $A$ and diffusion $B_{T}$, as function of charm momentum at fixed $T=0.2 \mathrm{GeV}$ including now the $\gamma^{*} / M=0.75$ case. If we can see that considering bigger widths for Breit-Wigner distribution with respect to DQPM one, there is a decrease of the transport coefficients. Furthermore, a limited improvement for FDT validity is observed for the case of larger widths where the FDT is satisfied within 10 $\%$, as shown by open green circles in Fig. 8. Finally, in Fig. 9 it is shown the $A(p) / \epsilon$ ratio as a function of the charm quark momentum and for temperature $T=0.2 \mathrm{GeV}$. Comparing black solid line with red dashed line, we observe a scaling between on-shell and off-shell calculation for $p \geq 2-3 \mathrm{GeV}$ and breaking a lower momentum. This suggests that for the widths used in DQPM the difference in the drag coefficient in off-shell case is completely re-adsorbed for high momentum of charm. Furthermore, if we increase the widths as shown by open green circles we get that the drag coefficient shows a larger breaking of the scaling at least for $p \lesssim 2-3 \mathrm{GeV}$ that at $p \rightarrow 0$ is maximal and corresponds to a reduction of about a $40 \%$.

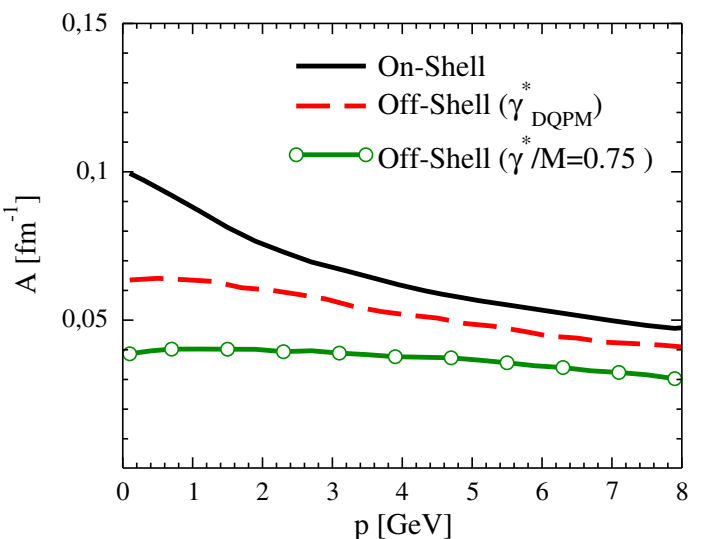

Fig. 6 Drag coefficient $(A)$ as a function of momentum for fixed medium temperature at $T=0.2 \mathrm{GeV}$ for on-shell approach (black solid line) and off-shell approach (red dashed line). The open green circles refers to the case with larger widths with fixed ratio $\gamma_{i}^{*} / M_{i}=0.75$

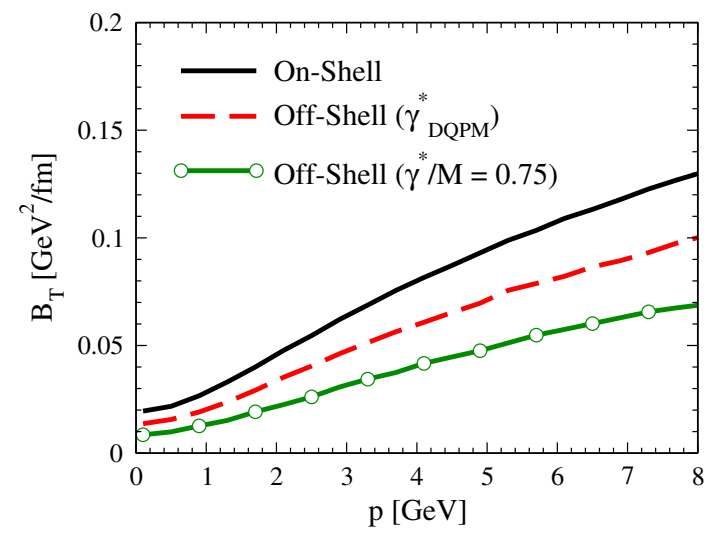

Fig. 7 Diffusion coefficient $\left(B_{T}\right)$ as a function of momentum and for fixed medium temperature at $T=0.2 \mathrm{GeV}$ for on-shell approach (black solid line) and off-shell approach (red dashed line). The open green circles refers to the case with larger widths with fixed ratio $\gamma_{i}^{*} / M_{i}=$ 0.75

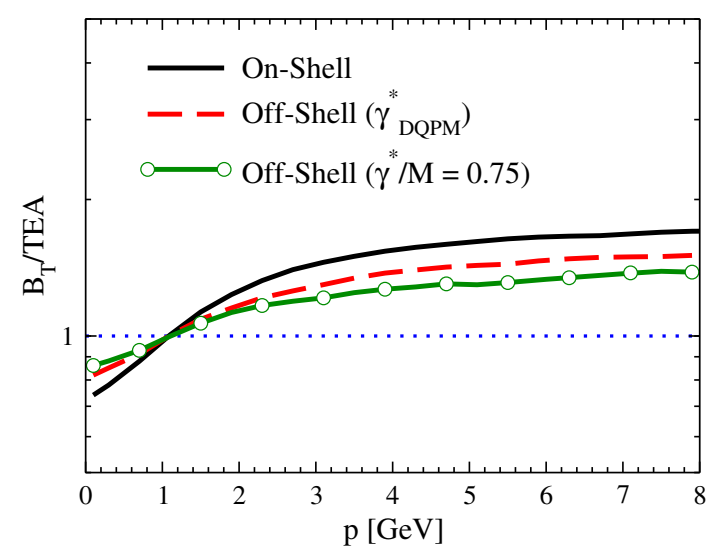

Fig. $8 B_{T} / T E A$ as a function of charm momentum and fixed temperature $T=0.2 \mathrm{GeV}$ for both on-shell and off-shell approaches. Same legend as in Fig. 6 


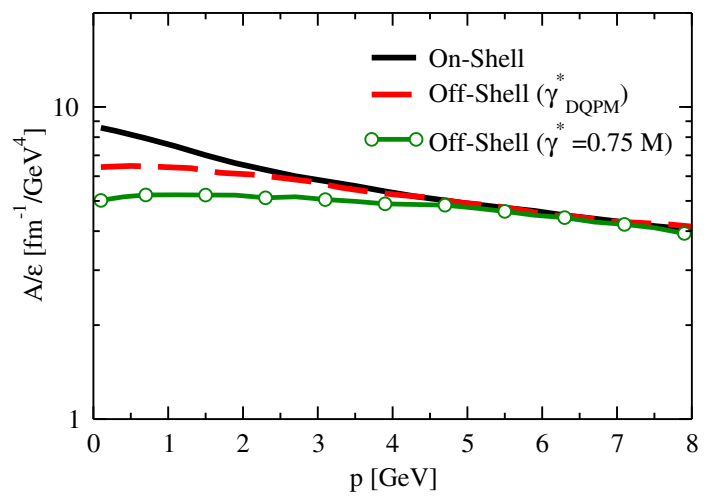

Fig. $9 A(p) / \epsilon$ ratio as function of charm momentum at $T=0.2 \mathrm{GeV}$ for on shell (black solid line) and off-shell $\gamma_{D Q P M}^{*}$ (red dashed line) and $\gamma^{*}=0.75 M$ (open green circles)

The results obtained for the drag and diffusion coefficients are in agreement with the results obtained within the DQPM and in the intermediate momentum range are similar to the one obtained within the TAMU approach, see $[7,8]$.

\section{Heavy quarks momentum evolution in the QGP: on- and off-shell Boltzmann and Langevin dynamics}

In this section we discuss about the time evolution of HQs within Boltzmann scattering with on-shell quarks and gluons and the extension of the Boltzmann collision integral to account for off-shell conditions by mean of the Breit-Wigner spectral functions as done for the transport coefficients, see Eq. 20. We are interested in the evolution of the HQ distribution function $f_{Q}(x, p)$ in a thermal bulk described through QPM approach and we have considered a plasma in equilibrium in a box with constant temperature T. In this study, the starting point to investigate the HQ evolution for both on-shell and off-shell approaches is the simplified form of the Boltzmann equation that is expressed in Eq. 2. We can write:

$\frac{\partial f_{Q}}{\partial t}=\frac{1}{E_{Q}} C\left[\hat{f}_{q}, \hat{f}_{g}, f_{Q}\right]$.

Since in the previous equation the field gradients are discarded, it is valid for both on-shell and off-shell dynamics, with the last embedded in $C\left[\hat{f}_{q}, \hat{f}_{g}, f_{Q}\right]$ according to Eq. 20. After a time discretization the Boltzmann equation can be written as

$f(t+\Delta t, p)=f(t, p)+\frac{\Delta t}{E_{Q}} C[f]+O\left(\Delta t^{3}\right)$.

As for the transport coefficients, the numerical solution of Boltzmann equation is obtained by a code that implement a Monte-Carlo integration method for the full collision kernel described by Eqs. 3 and 20. Different tests have been done

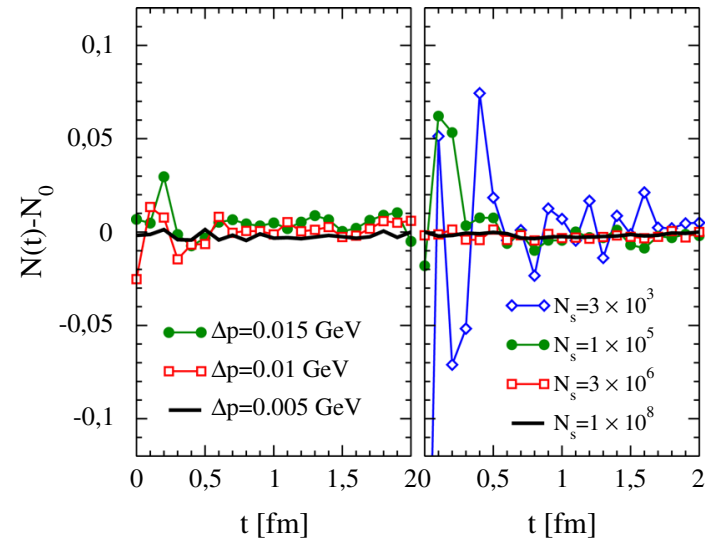

Fig. $10 N(t)-N_{0}$ as a function of time. Left panel different lines are for different momentum discretization with a fixed number of MC sampling to $N_{s}=10^{8}$ while in the right panel the different lines refers to the different MC sampling used for $\Delta P=0.005 \mathrm{GeV}$

in order to verify the convergency of the collision integrals both in on-shell and off-shell case. It is important to fix the number of MonteCarlo samples $N_{s}$, in particular for off-shell case, where we have two additional integrations over spectral function that give the weight of each initial and final mass of light partons in the bulk. In this study, we have discretized the time and the HQ momentum $p$ in the propagation in order to calculate the evolution of phase-space distribution function of charm quarks. We want that the integral over the distribution function is conserved. Therefore, we can write:

$\frac{\partial N}{\partial t}=\int d^{3} p \frac{\partial f}{\partial t}=\int d^{3} p \frac{C[f]}{E_{Q}} \equiv \bar{C}$

where $N$ is the number of charms quarks. If the integral is not conserved, we can assume a variation $\Delta N$ according to $N(t)=N_{0}+\bar{C} \Delta t$ where $N_{0}$ is the initial number of charm quarks.

In Fig. 10 it is shown an example of study of the convergence of the off-shell collision integral, similar results we get also for the on-shell case. In particular, we have studied the time evolution of $\Delta N$ for different momentum discretization $\Delta p$ (left panel) and number of samples $N_{s}$ (right panel) used for the Monte-Carlo calculation of the collision integral. We have found that the most appropriate number of momentum discretization and Monte-Carlo samples is $\Delta p=5 \times 10^{-3}$ $\mathrm{GeV}$ and $N_{s}=10^{8}$. A similar study it has been performed for the time step $\Delta t$ we found that $\Delta t=0.1 \mathrm{fm}$ is enough to get the convergency of differential equation Eq. 27. Within the numerical approach used in this paper both particle number and energy are conserved to an accuracy better than $10^{-4}$ within the time range explored in the following figures. We have also checked that at the thermalization time $\tau_{\text {eq. }}$, the distribution reaches the equilibrium condition defined by Juttner-Boltzmann solution and the integral over distribution function is conserved at each time step. 
In soft scattering approximation, another standard approach used to describe the HQ propagation in the bulk medium of quarks and gluons is by means of a Fokker-Planck equation of Eq. 11. The Fokker-Planck equation is solved by a stochastic differential equation given by the Langevin equation where the equations of motion of the HQs are given by

$d x_{i}=\frac{p_{i}}{E} d t$

$d p_{i}=-A p_{i} d t+C_{i, j} \rho_{j} \sqrt{d t}$.

This set of equations describe the variation of coordinate $d x_{i}$ and momentum $d p_{i}$ in each time step $d t[14,55,56]$. In the previous equation, $A$ represents drag force and $C_{i, j}$ is the covariance matrix that describes stochastic force in term of independent Gaussian-normal distributed random variables $\rho_{j}$. The random variable $\rho_{j}$ obey to the following distribution $p(\rho)=(2 \pi)^{-3 / 2} e^{-\rho^{2} / 2}$ with the conditions that $\left\langle\rho_{i}\right\rangle=0$ and $\left\langle\rho_{i} \rho_{j}\right\rangle=\delta\left(t_{i}-t_{j}\right)$. This covariance matrix is related to diffusion coefficient in the following way:

$C_{i, j}=\sqrt{2 B_{T}} P_{i, j}^{\perp}+\sqrt{2 B_{L}} P_{i, j}^{\|}$

where $B_{T}$ and $B_{L}$ are respectively the transverse and longitudinal component of diffusion coefficient. In general $B_{L}=$ $B_{T}=D$ for $p \rightarrow 0$ and it is a standard choice by several groups also at finite momenta $p$ when studying the HQ observables in realistic simulation of ultra-relativistic collisions [12-14,56]. In Langevin approach, the fluctuationdissipation relation $B_{T}=T E A$ is commonly employed even if a microscopic derivation in general violates such relation at finite momentum as we have discussed in the previous sections. We have verified that the numerical solution of Langevin equation converges to the equilibrium solution $f_{e q}=e^{-E / T}$ at very large time. In order to fulfill this condition we reformulate the fluctuation-dissipation theorem as suggested by the pre-Ito interpretation [55] and we solve the Langevin equation with the condition:

$A(p)=\frac{D(p)}{E T}-\frac{D^{\prime}(p)}{p}$

therefore taking $D(p)=B_{T}(p)$ as calculated by scattering matrix according to Eqs. 15 and 18 and we evaluate the correct drag force to achieve equilibrium distribution at themalization time. This procedure is necessary to guarantee that for $t \rightarrow \infty\left(\gtrsim \tau_{e q}.\right)$ also the Langevin approach converges to the correct equilibrium distribution as naturally occurs for the Boltzmann evolution. Such agreement is shown in the right-low panel of Fig. 11.

\subsection{Results on HQ moment evolution}

To investigate the differences between the heavy quark dynamics implied by Boltzmann on-shell dynamics and offshell dynamics, we study the heavy quark time evolution of

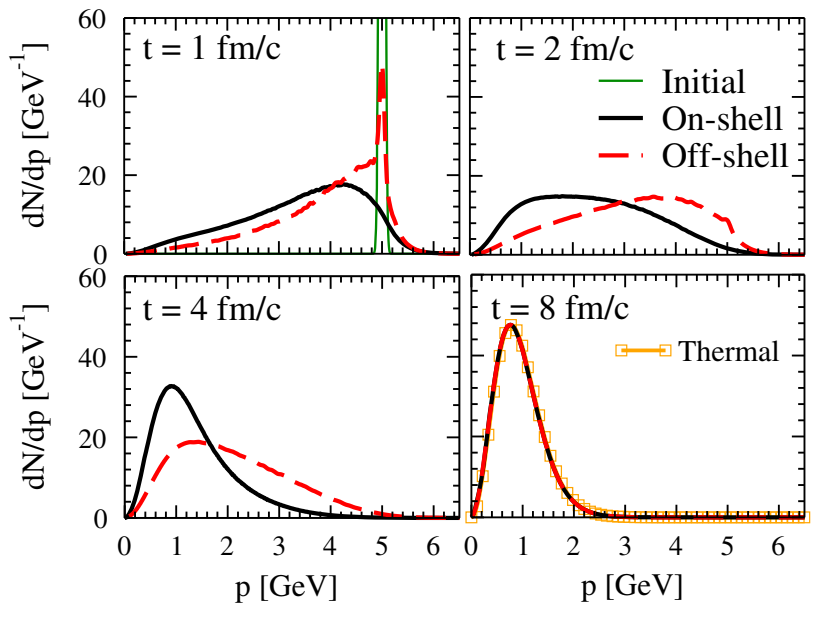

Fig. 11 Charm quark momentum distribution as a function of the charm quark momentum at four different times $t=1 \mathrm{fm} / \mathrm{c}$ (left upper panel) and $t=2 \mathrm{fm} / \mathrm{c}$ (right upper panel), $t=4 \mathrm{fm} / \mathrm{c}$ (left lower panel) and $t=8 \mathrm{fm} / \mathrm{c}$ (right lower panel). Black solid lines are for on-shell dynamics while red dashed lines are for off-shell dynamics. The solid thin green line is the initial distribution that is the same for both calculations

the momentum distribution. In the following results we have considered a thermal bulk of light quarks and gluons at a temperature of $T=0.2 \mathrm{GeV}$. In our calculation the initial charm quark distribution is assumed as an approximately delta distribution at $p_{0}=5 \mathrm{GeV}$ shown by the green line in the left panel of Fig. 11.

In Fig. 11, we show the time evolution of the momentum distribution $d N / d p$ for both on-shell (black solid line) and off-shell dynamics (red dashed line) with $\gamma_{D O P M}^{*}$. As shown the Boltzmann approach with off-shell collision integral has a slower dynamics than the on-shell one. This can be understood as due to the fact that in the off-shell case the spreading of the bulk mass according to the quarks and gluons spectral functions can be assimilated as a system with a larger average effective mass, considering that the part of the spectral function at larger mass has anyway a larger phase space. At $t>4 \mathrm{fm} / \mathrm{c}$ for both cases the momentum distribution tend towards a thermal distribution at $T=0.2 \mathrm{GeV}$ as shown in the right lower panel of Fig. 11 by the open square points. The main difference is a faster evolution for the on-shell case that is however mainly due to the fact that the on-shell and off shell dynamics have an underlying bulk system with a different energy density and the drag coefficients are those corresponding to Fig. 9.

In the following discussion we will show instead two different calculations for two different drag and diffusion coefficient implementation. The motivation is twofold. From one hand we try to discard the pure off-shell effect in the HQ dynamics from the on-shell one. From the other hand we are motivated by the fact that different approaches have been used to extract the HQ transport coefficients from the com- 


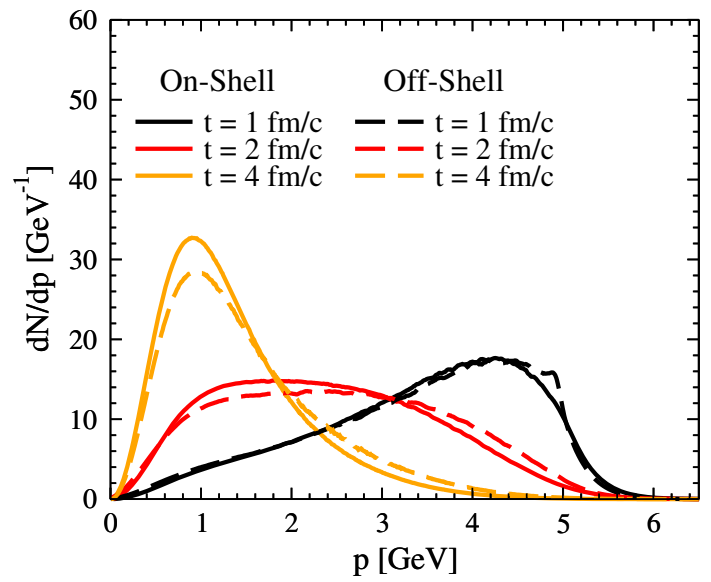

Fig. 12 Time evolution of the charm quark momentum distribution in a thermal bulk at $T=0.2 \mathrm{GeV}$. Solid lines are for the on-shell dynamics while dashed lines are for off-shell dynamics. Different colors are for different times. For the off-shell case in this calculation $\gamma_{i}^{*} / M_{i}=0.75$ and the energy density of the bulk is the same to the on-shell case

parison of the observables, like nuclear modification factor and anisotropic flows, with the experimental data. In particular we will compare the results obtained within Langevin approach and within the on-shell and off-shell dynamics. We firstly have considered one case where we scale the drag coefficient of the off-shell kernel to the on-shell one by the energy density for the case at larger width considered $\gamma^{*} / M=0.75$.

Here we considered the evolution on-shell and off-shell $\left(\gamma^{*} / M=0.75\right)$ for a bulk that has been tuned to have the same energy density in agreement with $1 \mathrm{QCD}$ calculation. This case is different from the previous one because here the bulk QGP as the same energy density in the two cases. As was shown in the previous section in Fig. 9, the effect of the transport coefficient between off-shell and on-shell in large part due to the difference in the energy density is damped when considered the physical case where both on-shell and off-shell are tuned to the same energy density. In order to achieve this point, we upscale the off-shell scattering matrix by a constant factor $k=\epsilon_{\text {on-Shell }}(T) / \epsilon_{\text {off-Shell }}(T)$ that in our simulation for a thermal bulk at $T=0.2 \mathrm{GeV}$ is about $k \approx 1.5$ corresponding to an underlying increase of the coupling $g(T)$ of about a 6\%. In Fig. 12 it is shown the time evolution of the charm momentum distribution for both onshell and off-shell dynamics. We can notice that off-shell drag coefficient remains smaller than on-shell one especially at low momenta and this implies an off-shell dynamics that is slightly slower with respect to the on-shell case, but the effect remains quite small. From these calculation we can assert that the differences seen in Fig. 11 are mainly due to the different energy density induced by the fact that keeping equal the pole value of the mass and dressing the system by a finite width induces a decreasing of the energy of the system. Such an effect is nearly negligible for the off-shell

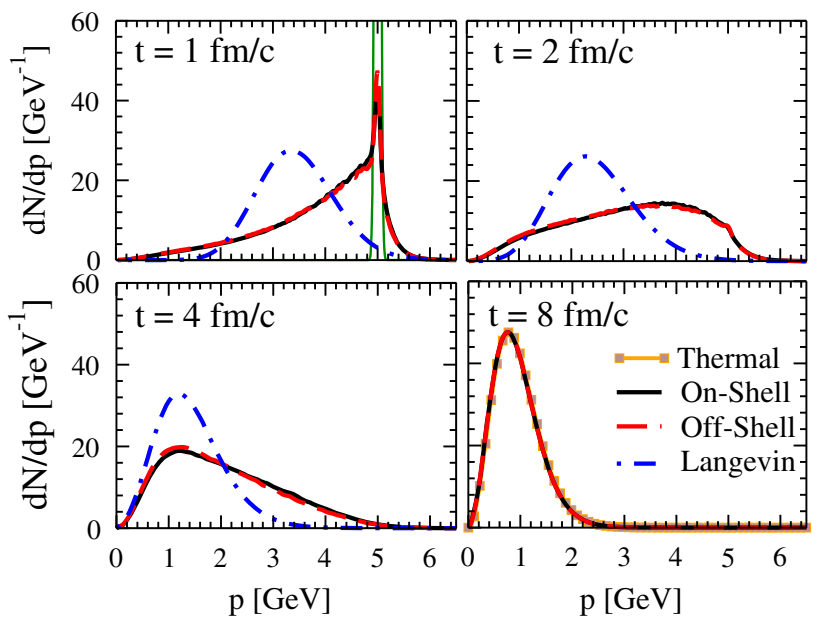

Fig. 13 Charm quark momentum distribution as a function of the charm quark momentum at four different times $t=1 \mathrm{fm} / \mathrm{c}$ (left upper panel) and $t=2 \mathrm{fm} / \mathrm{c}$ (right upper panel), $t=4 \mathrm{fm} / \mathrm{c}$ (left lower panel) and $t=8 \mathrm{fm} / \mathrm{c}$ (right lower panel). Black solid lines are for on-shell dynamics, red dashed lines are for off-shell dynamics while blue dash-dotted lines are for langevin dynamics. The solid thin green line is the initial distribution that is the same for each calculations

case with $\gamma_{D Q P M}^{*} \simeq 0.3-0.4 M$, but becomes sizeable for $\gamma^{*} / M=0.75$. However the pure off-shell dynamics does not show relevant differences as we can see comparing the on-shell (solid lines) and off-shell mode (dashed lines). This suggests that on-shell Boltzmann equation is still a quite good approximation to study the evolution of the charm momentum distribution at least up to $\gamma^{*}<M$.

Finally, we have performed another calculation where we upscale the on-shell scattering matrix $\left|\mathcal{M}_{Q}\right|^{2}$ in order to reproduce the same drag coefficient obtained with the offshell collision integral. This corresponds to multiply the onshell scattering matrix $\left|\mathcal{M}_{Q}\right|^{2}$ by a function $k(p)$. It may be considered as non realistic case because we have seen that the impact of off-shell dynamics on transport coefficient is momentum dependent and leads to induce a slower increase of the drag coefficient at lower momenta. We have considered it to study theoretically what happens if the interaction is such to generate exactly the same drag $A(p)$ at each momentum. We show the results of this set-up for the case $\gamma_{D Q P M}$. in Fig. 13. We can see that the time evolution of the HQ momentum distribution for the three different approaches onshell Boltzmann (solid lines), off-shell Boltzmann (dashed lines) and Langevin (dot-dashed lines). By comparing solid lines and dashed lines once where one impose the same drag coefficient the two approaches show the same evolution. Notice that in the Langevin calculations, we have used the pre-ito prescription where the diffusion coefficient is the one obtained within off-shell calculation shown in Fig. 7. As shown the Langevin dynamics consists of a shift of the average momenta with a fluctuation around it. This include the possibility that HQ obviously lose energy moving the 


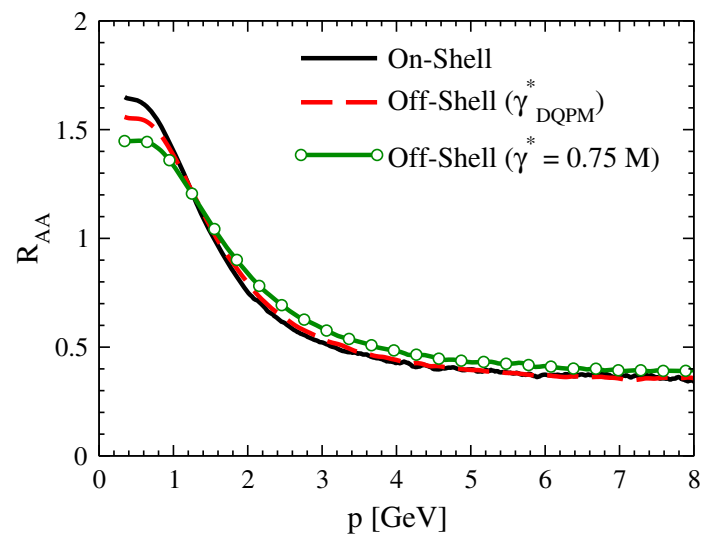

Fig. 14 Nuclear modification factor, $R_{A A}$ as function of charm momentum $p$. Solid lines refers to the case of on-shell calculations. The red dashed line refer to off-shell calculation with scattering matrix scaled with the energy density while the green open circles refer to the same calculation but with larger width fixed to $\gamma_{i}^{*} / M_{i}=0.75$

distribution to lower momenta but at the same time they can gain energy from the bulk producing a tail with momentum larger than the initial HQ momentum $p_{0}$. As shown, by the comparison between the Langevin and Boltzmann dynamics, the Boltzmann evolution of the charm quarks momentum does not have a Gaussian shape like one for the Langevin approach. Where at the initial time the Boltzmann dynamics with respect to the Langevin one shows at initial stages a larger contribution from the gain term in the collision integral with a global shape that is far from the Gaussian shape typical of Brownian motion [57].

\subsection{Nuclear modification factor $R_{A A}$ in Boltzmann and off-shell dynamics}

One of the main HQ observable investigated at RHIC and LHC energies is nuclear modification factor $R_{A A}$. It expresses the effective energy loss in nucleus-nucleus collision with respect to the production in proton-proton collisions. In general, $R_{A A}$ gives a quantitative estimate of heavy quarks-bulk interaction. Motivated by the phenomenological point of view, we have studied the impact of the results shown in the previous section on the evolution of the spectra in terms of the $R_{A A}(p)$ for charm quarks. We evaluate the Nuclear Modification factor using the charm quark distribution function at $t=t_{0}$ and $t=t_{f}$ as $R_{A A}=f_{C}\left(p, t_{f}\right) / f_{C}\left(p, t_{0}\right)$ both for on-shell and off-shell dynamics. In these calculation for the initial momentum distribution of charm quark, we have used the charm quark production in fixed order + nextto-leading log (FONLL) [58] which describes the D-meson spectra in proton-proton collisions after fragmentation.

In Fig. 14 we show the nuclear modification factor $R_{A A}$ for $t_{f}=4 \mathrm{fm} / \mathrm{c}$ as a function of the charm quark momentum for both on-shell and off-shell. Black Solid line refers to the case of on-shell calculations while the red dashed line and the green open circles refer to off-shell calculation with scattering matrix scaled to the case of the on-shell energy density and with $\gamma_{D Q P M}^{*}$ and $\gamma_{i}^{*} / M_{i}=0.75$ respectively. As shown, by comparing solid and dashed line, in the off-shell approach with $\gamma_{D Q P M}^{*}$ the nuclear modification factor $R_{A A}$ does not show significant difference with respect to on-shell calculations especially for intermediate and high momentum of quark charm as shown in the same condition for evolution of distribution function. Also for the case of off-shell dynamics with $\gamma^{*} / M_{i}=0.75$ we find that the $R_{A A}(p)$ is slightly larger at high $p$ than on-shell one and it differs from the on-shell calculation less than $10 \%$. Therefore a main result of thi work is that the off-shell dynamics does not modify significantly the relation between $R_{A, A}(p)$ and $D_{S}(T)$ and it would not represent a main source of uncertainty in the phenomenological determination of the space diffusion coefficient that are currently more dependent on the hadronization mechanism, Langevin versus Boltzmann transport equation, assumption for bulk QGP expansion, effects of non-equilibrium in the initial stage $[7,8,59]$.

\section{Summary and conclusion}

We have studied the impact of off-shell dynamics on the drag and diffusion transport coefficients. We have found that if one just include the off-shell dynamics of quasi-particles associated to a finite mass width this induce a moderate decrease of the density of the system an this leads to a smaller drag and diffusion charm coefficient that is dependent on charm momentum. However when the comparison is done renormalizing the energy density of the system, that is the one of lattice QCD, one can see that the main effect of off-shell dynamics is to reduce the increase of the drag $A(p, T)$ and $B_{T}(p, T)$ at lower momenta $p \lesssim 2-3 \mathrm{GeV}$. Such a reduction depends of the width and is maximal at $p=0$ being for $\gamma_{D Q P M} \approx 0.3-0.4 m_{q, g}$ about a $25 \%$ while increasing up to about a $35 \%$ for the $\gamma^{*}<0.75 m_{q, g}$. In both case at $p>3$ $\mathrm{GeV}$ such a difference disappears completely.

We then have studied how a charm of momentum $p$ loose energy in a bulk QGP in equilibrium at temperature $\mathrm{T}=$ $0.2 \mathrm{GeV}$, comparing for the first time the time evolution of the momenta in a Langevin, Boltzmann on-shell and Boltzmann off-shell transport approach. We find that at least in the regime of widths $\gamma^{*}<M$ the evolution of charm momenta are only slightly modified by off-shell dynamics, also the impact of the last on $R_{A A}(p)$ is of about a $5 \%$ at least at momenta $p>1 \mathrm{GeV}$. Therefore from a phenomenological point of view the relation between the nuclear modification factor $R_{A A}\left(p_{T}\right)$ and the space diffusion coefficient (or the drag) is not significantly modified by off-shell dynamics. 
Acknowledgements S. P., M. L. S., and V. G. acknowledge the support of INFN-SIM national project and linea di intervento 2, DFA-Unict. S. P. , M. L. S., and V. G. acknowledge the stimulating discussions and comments with G. Coci. This work was supported by the European Union's Horizon 2020 research and innovation program Strong 2020 under grant agreement No 824093 .

Data Availability Statement This manuscript has no associated data or the data will not be deposited. [Authors' comment: This is a theoretical paper and there is no data deposited. The data can be provided when asked.]

Open Access This article is licensed under a Creative Commons Attribution 4.0 International License, which permits use, sharing, adaptation, distribution and reproduction in any medium or format, as long as you give appropriate credit to the original author(s) and the source, provide a link to the Creative Commons licence, and indicate if changes were made. The images or other third party material in this article are included in the article's Creative Commons licence, unless indicated otherwise in a credit line to the material. If material is not included in the article's Creative Commons licence and your intended use is not permitted by statutory regulation or exceeds the permitted use, you will need to obtain permission directly from the copyright holder. To view a copy of this licence, visit http://creativecomm ons.org/licenses/by/4.0/.

Funded by SCOAP ${ }^{3}$.

\section{References}

1. B. Svetitsky, Phys. Rev. D 37, 2484 (1988). https://doi.org/10. 1103/PhysRevD.37.2484

2. G.D. Moore, D. Teaney, Phys. Rev. C 71, 064904 (2005). https:// doi.org/10.1103/PhysRevC.71.064904

3. X. Dong, V. Greco, Prog. Part. Nucl. Phys. 104, 97 (2019). https:// doi.org/10.1016/j.ppnp.2018.08.001

4. F. Prino, R. Rapp, J. Phys. G43(9), 093002 (2016). https://doi.org/ 10.1088/0954-3899/43/9/093002

5. A. Andronic et al., Eur. Phys. J. C 76(3), 107 (2016). https://doi. org/10.1140/epjc/s10052-015-3819-5

6. G. Aarts et al., Eur. Phys. J. A 53(5), 93 (2017). https://doi.org/10. 1140/epja/i2017-12282-9

7. S. Cao et al., Phys. Rev. C 99(5), 054907 (2019). https://doi.org/ 10.1103/PhysRevC.99.054907

8. A. Beraudo et al., Nucl. Phys. A 979, 21 (2018). https://doi.org/ 10.1016/j.nuclphysa.2018.09.002

9. S.K. Das, F. Scardina, S. Plumari, V. Greco, Phys. Rev. C 90, 044901 (2014). https://doi.org/10.1103/PhysRevC.90.044901

10. V. Greco, Nucl. Phys. A 967, 200 (2017). https://doi.org/10.1016/ j.nuclphysa.2017.06.044

11. H. van Hees, R. Rapp, Phys. Rev. C 71, 034907 (2005). https://doi. org/10.1103/PhysRevC.71.034907

12. H. van Hees, V. Greco, R. Rapp, Phys. Rev. C 73, 034913 (2006). https://doi.org/10.1103/PhysRevC.73.034913

13. H. van Hees, M. Mannarelli, V. Greco, R. Rapp, Phys. Rev. Lett. 100, 192301 (2008). https://doi.org/10.1103/PhysRevLett. 100.192301

14. S. Cao, S.A. Bass, Phys. Rev. C 84, 064902 (2011). https://doi.org/ 10.1103/PhysRevC.84.064902

15. G.Y. Qin, H. Petersen, S.A. Bass, B. Muller, Phys. Rev. C 82, 064903 (2010). https://doi.org/10.1103/PhysRevC.82.064903

16. S.K. Das, V. Chandra, J.E. Alam, J. Phys. G 41, 015102 (2013). https://doi.org/10.1088/0954-3899/41/1/015102

17. S.K. Das, J.E. Alam, P. Mohanty, Phys. Rev. C 82, 014908 (2010). https://doi.org/10.1103/PhysRevC.82.014908
18. W. Alberico, A. Beraudo, A. De Pace, A. Molinari, M. Monteno, M. Nardi, F. Prino, M. Sitta, Eur. Phys. J. C 73, 2481 (2013). https:// doi.org/10.1140/epjc/s10052-013-2481-z

19. M. He, R.J. Fries, R. Rapp, Phys. Rev. C 86, 014903 (2012). https:// doi.org/10.1103/PhysRevC.86.014903

20. M. He, R.J. Fries, R. Rapp, Phys. Lett. B 735, 445 (2014). https:// doi.org/10.1016/j.physletb.2014.05.050

21. Y. Xu, J.E. Bernhard, S.A. Bass, M. Nahrgang, S. Cao, Phys. Rev. C 97(1), 014907 (2018). https://doi.org/10.1103/PhysRevC. 97.014907

22. P. Gossiaux, J. Aichelin, Phys. Rev. C 78, 014904 (2008). https:// doi.org/10.1103/PhysRevC.78.014904

23. P. Gossiaux, R. Bierkandt, J. Aichelin, Phys. Rev. C 79, 044906 (2009). https://doi.org/10.1103/PhysRevC.79.044906

24. S. Ghosh, S.K. Das, S. Sarkar, J.E. Alam, Phys. Rev. D 84, 011503 (2011). https://doi.org/10.1103/PhysRevD.84.011503

25. J. Uphoff, O. Fochler, Z. Xu, C. Greiner, Phys. Lett. B 717, 430 (2012). https://doi.org/10.1016/j.physletb.2012.09.069

26. J. Uphoff, O. Fochler, Z. Xu, C. Greiner, Phys. Rev. C 84, 024908 (2011). https://doi.org/10.1103/PhysRevC.84.024908

27. T. Song, H. Berrehrah, D. Cabrera, J.M. Torres-Rincon, L. Tolos, W. Cassing, E. Bratkovskaya, Phys. Rev. C 92(1), 014910 (2015). https://doi.org/10.1103/PhysRevC.92.014910

28. S. Cao, T. Luo, G.Y. Qin, X.N. Wang, Phys. Rev. C 94(1), 014909 (2016). https://doi.org/10.1103/PhysRevC.94.014909

29. S. Cao, T. Luo, G.Y. Qin, X.N. Wang, Phys. Lett. B 777, 255 (2018). https://doi.org/10.1016/j.physletb.2017.12.023

30. B.I. Abelev et al., Phys. Rev. Lett. 98, 192301 (2007). https://doi.org/10.1103/PhysRevLett.106.159902. https://doi. org/10.1103/PhysRevLett.98.192301. [Erratum: Phys. Rev. Lett.106,159902(2011)]

31. J. Adam et al., JHEP 03, 081 (2016). https://doi.org/10.1007/ JHEP03(2016)081

32. S.S. Adler et al., Phys. Rev. Lett. 96, 032301 (2006). https://doi. org/10.1103/PhysRevLett.96.032301

33. A. Adare et al., Phys. Rev. Lett. 98, 172301 (2007). https://doi.org/ 10.1103/PhysRevLett.98.172301

34. B.B. Abelev et al., Phys. Rev. C 90(3), 034904 (2014). https://doi. org/10.1103/PhysRevC.90.034904

35. S.K. Das, J.E. Alam, P. Mohanty, Phys. Rev. C 80, 054916 (2009). https://doi.org/10.1103/PhysRevC.80.054916

36. W. Alberico, A. Beraudo, A. De Pace, A. Molinari, M. Monteno, M. Nardi, F. Prino, Eur. Phys. J. C 71, 1666 (2011). https://doi.org/ 10.1140/epjc/s10052-011-1666-6

37. T. Lang, H. van Hees, J. Steinheimer, G. Inghirami, M. Bleicher, Phys. Rev. C 93(1), 014901 (2016). https://doi.org/10.1103/ PhysRevC.93.014901

38. T. Song, H. Berrehrah, D. Cabrera, W. Cassing, E. Bratkovskaya, Phys. Rev. C 93(3), 034906 (2016). https://doi.org/10.1103/ PhysRevC.93.034906

39. S. Cao, G.Y. Qin, S.A. Bass, Phys. Rev. C 92(2), 024907 (2015). https://doi.org/10.1103/PhysRevC.92.024907

40. S.K. Das, F. Scardina, S. Plumari, V. Greco, Phys. Lett. B 747, 260 (2015). https://doi.org/10.1016/j.physletb.2015.06.003

41. S.K. Das, M. Ruggieri, F. Scardina, S. Plumari, V. Greco, J. Phys. G 44(9), 095102 (2017). https://doi.org/10.1088/1361-6471/aa815a

42. Y. Sun, G. Coci, S.K. Das, S. Plumari, M. Ruggieri, V. Greco, Phys. Lett. B 798, 134933 (2019). https://doi.org/10.1016/j.physletb. 2019.134933

43. R. Katz, C.A.G. Prado, J. Noronha-Hostler, J. Noronha and A.A.P. Suaide, Phys. Rev. C 102(2), 024906 (2020). https://doi.org/10. 1103/PhysRevC.102.024906

44. S. Plumari, G. Coci, V. Minissale, S.K. Das, Y. Sun, V. Greco, Phys. Lett. B 805, 135460 (2020). https://doi.org/10.1016/j.physletb. 2020.135460 
45. H. Berrehrah, E. Bratkovskaya, W. Cassing, P. Gossiaux, J. Aichelin, M. Bleicher, Phys. Rev. C 89(5), 054901 (2014). https://doi. org/10.1103/PhysRevC.89.054901

46. T. Song, H. Berrehrah, J.M. Torres-Rincon, L. Tolos, D. Cabrera, W. Cassing, E. Bratkovskaya, Phys. Rev. C 96(1), 014905 (2017). https://doi.org/10.1103/PhysRevC.96.014905

47. S. Cao, K.J. Sun, S.Q. Li, S.Y.F. Liu, W.J. Xing, G.Y. Qin, C.M. Ko, Phys. Lett. B 807, 135561 (2020). https://doi.org/10.1016/j. physletb.2020.135561

48. M. Ruggieri, F. Scardina, S. Plumari, V. Greco, Phys. Rev. C 89(5), 054914 (2014). https://doi.org/10.1103/PhysRevC.89.054914

49. S. Plumari, G.L. Guardo, F. Scardina, V. Greco, Phys. Rev. C 92(5), 054902 (2015). https://doi.org/10.1103/PhysRevC.92.054902

50. S. Plumari, Eur. Phys. J. C 79(1), 2 (2019). https://doi.org/10.1140/ epjc/s10052-018-6510-9

51. S. Plumari, W.M. Alberico, V. Greco, C. Ratti, Phys. Rev. D 84, 094004 (2011). https://doi.org/10.1103/PhysRevD.84.094004

52. W. Cassing, E. Bratkovskaya, Nucl. Phys. A 831, 215 (2009). https://doi.org/10.1016/j.nuclphysa.2009.09.007

53. E. Bratkovskaya, W. Cassing, V. Konchakovski, O. Linnyk, Nucl. Phys. A 856, 162 (2011). https://doi.org/10.1016/j.nuclphysa. 2011.03.003
54. H. Berrehrah, P.B. Gossiaux, J. Aichelin, W. Cassing, E. Bratkovskaya, Phys. Rev. C 90(6), 064906 (2014). https://doi.org/ 10.1103/PhysRevC.90.064906

55. R. Rapp, H. van Hees 111-206 (2010). https://doi.org/10.1142/ 9789814293297_0003. e-Print: arXiv:0903.1096 [hep-ph]

56. G.D. Moore, D. Teaney, Phys. Rev. C 71, 6 (2005). https://doi.org/ 10.1103/physrevc.71.064904

57. F. Scardina, D. Perricone, S. Plumari, M. Ruggieri, V. Greco, Phys. Rev. C 90(5), 054904 (2014). https://doi.org/10.1103/PhysRevC. 90.054904

58. M. Cacciari, S. Frixione, N. Houdeau, M.L. Mangano, P. Nason, G. Ridolfi, JHEP 10, 137 (2012). https://doi.org/10.1007/ JHEP10(2012)137

59. Y. Xu et al., Phys. Rev. C 99(1), 014902 (2019). https://doi.org/10. 1103/PhysRevC.99.014902 